\title{
The mirror mechanism in schizophrenia spectrum disorders: Protocol for a systematic review and meta-synthesis
}

Amir Valizadeh ( $\nabla$ thisisamirv@gmail.com )

Tehran University of Medical Sciences https://orcid.org/0000-0001-5983-8527

Nazanin Hedayati Amlashi

Tehran University of Medical Sciences https://orcid.org/0000-0003-0804-0018

Anita Rasooli

Tehran University of Medical Sciences https://orcid.org/0000-0002-5963-1419

Mathew Mbwogge

London School of Hygiene and Tropical Medicine https://orcid.org/0000-0003-0594-1937

Ainaaz Haadi

Tehran University of Medical Sciences https://orcid.org/0000-0002-6613-1877

\section{Systematic Review}

Keywords: Schizophrenia, Mirror Neurons

Posted Date: February 25th, 2021

DOI: https://doi.org/10.21203/rs.3.rs-264432/v4

License: (c) (1) This work is licensed under a Creative Commons Attribution 4.0 International License. Read Full License 


\section{Abstract}

Introduction: Mirror neurons are visuomotor neurons that perform mirror mechanisms, meaning each time an individual observes another individual performing an action, these neurons which encode that action, are activated in the observer's cortical motor system. One of the cognitive domains of controversy in schizophrenia is Motor Resonance, also known as the Mirror Neuron System (MNS). In the current paper, we aim to review all the available literature regarding mirror mechanism examination in patients with schizophrenia and present an explicit summary of the available findings up to this date.

Methods: We will search MEDLINE (through PubMed), Embase, and Web of Science for papers which evaluated the functional integrity of MNS, using Electroencephalography (EEG), Magnetoencephalography (MEG), Transcranial magnetic stimulation (TMS), Functional magnetic resonance imaging (fMRI), near-infrared spectroscopy (NIRS), Eye-tracking, and muscle activation (EMG) in schizophrenia spectrum disorders. We will perform a meta-synthesis based on vote counting methods on the data. We will also evaluate the publication bias using Funnel plots and assess the confidence in cumulative evidence using CERQual.

\section{Introduction}

\section{Rationale}

Schizophrenia is one of the most debilitating and common neuropsychiatric disorders in the world, with an estimated incidence of $1 \%$ in the population worldwide (2). Deficits in a variety of cognitive domains are well-known for this disorder (3-5) and they are listed as specifiers for schizophrenia in the $11^{\text {th }}$ revision of the International Classification of Diseases (ICD) (6). One of the cognitive domains of controversy in schizophrenia is Motor Resonance, also known as the Mirror Neuron System (MNS). Mirror neurons are visuomotor neurons that perform mirror mechanisms, meaning each time an individual observes another individual performing an action, these neurons which encode that action, are activated in the observer's cortical motor system $(7,8)$. These neurons were first discovered in the premotor area F5 of macaque monkeys (9-12). Later, similar neurons were found in the inferior parietal lobule, area PF, of macaque monkeys and the concept of 'mirror system' was established. Since the discovery of mirror neurons, some studies have claimed the discovery of similar neurons in various regions of the human brain, including the ventral premotor cortex $(13,14)$, inferior frontal gyrus (15-19), and inferior parietal lobule $(14,20)$. Meta-analyses of $\mathrm{fMRI}$ studies have demonstrated that there might be other brain regions too with mirror neurons including the dorsal premotor cortex, superior parietal lobe, temporal gyrus, and cerebellum $(21,22)$.

Several important functions beyond the action domain have been theorized for the mirror neuron system (MNS). For example, it has been posited as a fundamental building block for understanding others' actions (23). It is suggested that the possible specific cognitive role of mirror neurons might be that of encoding the intentions of the actor $(8,24,25)$. Also, the act of imitation has been suggested to rely on 
MNS $(7,26,27)$. This idea seems eligible, considering that the parieto-frontal motor regions are known to be typically involved in the planning and execution of actions $(21,28-30)$. lacoboni has suggested a "core circuit" for imitation that includes three regions, two of which include parts of MNS (31), although because monkeys are relatively poor in the task of imitation has brought arguments in this regard. Considering these findings there is an idea that the direct goal encoding feature of MNS provides a primary mechanism both for understanding other people and for imitating them (32). More recently, researchers suggest that MNS may play a role in human infants' ability to map similarities between self and others, and thus may be involved in providing a foundation for social-cognitive development (33). Additionally, there has been emphasis' on ties between MNS and empathy $(34,35)$, and MNS and language $(36,37)$.

To date, there has never been a systematic review of the studies which examine mirror mechanism in patients with schizophrenia. Based on a recent narrative review in Nature Reviews journal (38), findings in this regard are mixed and not compatible. This stresses the necessity of a systematic examination of the studies in this subject. In the current paper, we aim to review all the available literature regarding mirror mechanism examination in patients with schizophrenia and present an explicit summary of the available findings up to this date.

\section{Objectives}

To investigate the mirror mechanism in patients with schizophrenia and related disorders (psychosis spectrum disorder) and present an explicit view of available findings regarding this manner up to the date.

\section{Methods}

Design and methods used for this protocol review comply with Centre for Reviews and Dissemination (CRD's) Guidance For Undertaking Reviews in Healthcare (39), Meta-analyses of Observational Studies in Epidemiology (MOOSE) (40) and is reported in line with Preferred Reporting Items for Systematic Reviews and Meta-Analysis Protocols (PRISMA-P) (1). Eligibility criteria were informed using the SPIDER (41) and MOOSE guidelines.

\section{Eligibility criteria}

(S) Sample: Adults of any age and sex with the diagnosis of schizophrenia, schizoaffective disorder, and psychosis spectrum disorder in general confirmed by a physician according to International Classification of Diseases (ICD) $(6,42,43)$ or Diagnostic and Statistical Manual of Mental Disorders (DSM) $(44,45)$ guidelines, irrespective of the severity of disease and duration of illness. Participants with any other confirmed structural or functional neurologic disorders will be excluded. 
(PI) Phenomenon of Interest: The mirror neuron system (MNS) functional integrity.

(D) Design: Observational cohort and cross-sectional studies.

(E) Evaluation: Electroencephalography (EEG), Magnetoencephalography (MEG), Transcranial magnetic stimulation (TMS), Functional magnetic resonance imaging (fMRI), near-infrared spectroscopy (NIRS), Eye-tracking, and muscle activation (EMG).

(R) Research type: Qualitative, quantitative, and mixed-methods research could be searched for.

\section{Information sources}

The search will employ sensitive topic-based strategies designed for each database with no time frame limitations. There will be no language or geographical restrictions either. We will perform our search on the $10^{\text {th }}$ of February, 2021.

Databases:

- MEDLINE through PubMed

- Embase

- Science Citation Index - Expanded (Web of Science)

- Conference Proceedings Citation Index - Science (Web of Science)

\section{Search strategy}

Our search strategies for all the databases included in our study, namely MEDLINE (through PubMed), Embase, Science Citation Index - Expanded (Web of Science), and Conference Proceedings Citation Index - Science (Web of Science) are presented in appendix A.

\section{Study records}

\section{Data management}

Records will be managed through EndNote version X9 (46); specific software for managing bibliographies. 


\section{Selection process}

Two reviewers $(\mathrm{NH}$ and $\mathrm{AH}$ ) will independently screen the title and abstract of identified studies for inclusion. We will link publications from the same study to avoid including data from the same study more than once. If any study cannot be clearly excluded based on its title and abstract, its full text will be reviewed. A study will be included when both reviewers independently assess it as satisfying the inclusion criteria from the full text. A third reviewer (AV) will act as arbitrator in the event of disagreement following discussion.

\section{Data collection process}

Using a standardized form, two reviewers (AR and MM) will extract the data independently. A third reviewer (AV) will independently check the data for consistency and clarity. We will attempt to extract data presented only in graphs and figures whenever possible but will include such data only if two reviewers independently obtain the same result. If studies are multi-center, then where possible we will extract data relevant to each. If necessary, we will attempt to contact study authors through an open-ended request to obtain missing information or for clarification.

\section{Data items}

Data extracted will include the following summary data: sample characteristics, sample size, type of modality used for examining MNS in participants, the task that was used for the study, founding sources, declarations of interests, results, and summary of the findings as either normal, abnormal, and mixed (indicating that different components of the data suggest different things, or that the reported results are not entirely statistically robust).

\section{Outcomes and prioritization}

Studies will be grouped according to the different modalities used, which may include Electroencephalography (EEG), Magnetoencephalography (MEG), Transcranial magnetic stimulation (TMS), Functional magnetic resonance imaging (fMRI), near-infrared spectroscopy (NIRS), Eye-tracking, and muscle activation (EMG). However, studies using general behavioral measures (e.g. imitation tasks) and studies of reaction time during automatic imitation will be excluded. In the end, we will review other approaches that did not fit in the standard categorization of tasks, and also the findings from structural MRI studies.

\section{Risk of bias in individual studies}


Two authors (AR and MM) will independently evaluate the included studies for risks of bias. We will discuss any disagreement and document our decisions, and a third author (AV) will act as arbitrator in such a case. Cohen's $\mathrm{k}$ will be used to assess agreement between reviewers. All tools and processes will be piloted before use. We will use the NIH Quality Assessment Tool for Observational Cohort and CrossSectional Studies (47). This tool consists of fourteen questions which will address the following: 1Research question, 2 and 3-Study population, 4- Study eligibility criteria, 5- Sample size justification, 6Whether the exposure assessed prior to outcome measurement, 7- Wether sufficient timeframe was given to see an effect, 8- Different levels of the exposure of interest, 9- Exposure measures and assessment, 10Repeated exposure assessment, 11- Outcome measures, 12- Blinding of outcome assessors, 13- Followup rate, and 14- Statistical analysis. There are five possible answers to each question: yes, no, cannot determine, not applicable, and not reported. Finally, there are three possible judgments for the quality rating of each study: high quality, fair quality, and low quality. A high risk of bias translates to a rating of poor quality, while a low risk of bias translates to a rating of good quality. The tool, with the authors' judgment for a "yes" answer to each question, is presented in Appendix B.

\section{Data synthesis}

We will use R version 4 (48) as the software for our data synthesis. A meta-synthesis will be performed based on vote counting methods and results will be presented as a harvest plot. A summary of all studies included in the synthesis will also be presented. In this table we will present the following:

- Modality of the study (EEG, fMRI, etc.)

- Study ID

- Number of schizophrenia participants

- Mean age of participants (in years)

- Task: the task that was used with the modality.

- Results

- Summary: the results of each study will be summarized in terms of whether the paper provides evidence for an abnormal MNS in schizophrenia, a normal MNS, or evidence that is mixed. Mixed evidence can mean either that different components of the data suggest different things, or because the reported results are not entirely statistically robust.

\section{Meta-bias}

To evaluate the risk of reporting bias across studies, a test for funnel plot asymmetry will be conducted. This test examines whether the relationship between estimated effect size and study size is greater than 
chance (49). Funnel plots will be generated for visual inspection of potential publication bias. In the presence of publication bias, the plot will be symmetrical at the top, and data points will increasingly be missing from the middle to the bottom parts of the plot (50).

\section{Confidence in cumulative evidence}

The strength of the overall body of evidence will be assessed using the Confidence in Evidence from Reviews of Qualitative research method (CERQual) (51). This approach uses four components to evaluate confidence in the review findings. These include the methodological limitations of included studies, the relevance of the included studies to review questions, the coherence of the review findings, and the adequacy of the data that contributes to each review finding. In the first instance, MM will evaluate each finding using the four components of CERQual and a four-point scoring system ranging from 'no or very minor concerns' to 'substantial concerns'; AV then checks the evaluation. The review authors will meet and discuss the scores and assign each finding an overall CERQual assessment score. Each finding starts with a 'high confidence' score which could be downgraded to 'moderate confidence', 'low confidence', or 'very low confidence' if the CERQual process revealed concerns.

\section{Appendices}

\section{Appendix A}

\section{Embase}

\#1. 'mirror neuron system':ab,ti

\#2. 'mirror system':ab,ti

\#3. 'motor resonance':ab,ti

\#4. (neuron NEAR/5 mirror):ab,ti

\#5. (brain NEAR/5 mirror):ab,ti

\#6. 'mirror neuron'/exp

\#7. \#1 OR \#2 OR \#3 OR \#4 OR \#5 OR \#6

\#8. 'schizophrenia spectrum disorder'/exp

\#9. schizo*:ab,ti

\#10. (psychosis NEAR/5 spectrum):ab,ti 
\#11. \#8 OR \#9 OR \#10

\#12. \#7 AND \#11

\#13. \#12 AND [embase]/lim

\section{MEDLINE (through PubMed)}

(mirror neuron system[tw] OR Mirror Neurons[mh] OR mirror neuron*[tw] OR mirror system[tw] OR motor resonance[tw]) AND (schizo*[tw] OR Schizophrenia[mh] OR psychosis [tw])

\section{Web of Science}

\#1. TS="mirror neuron system"

\#2. TS="mirror system"

\#3. TS="motor resonance"

\#4. TS=(neuron NEAR/5 mirror)

\#5. TS=(brain NEAR/5 mirror)

\#6. \#1 OR \#2 OR \#3 OR \#4 OR \#5

\#7. TS=(schizo*)

\#8. TS=(psychosis NEAR/5 spectrum)

\#9. \#7 OR \#8

\#10. \#6 AND \#9

\section{Appendix B}

NIH Quality Assessment Tool for Observational Cohort and Cross-Sectional Studies (47) 


\begin{tabular}{|c|c|c|}
\hline Number & Question & Authors' judgment for "yes" \\
\hline \#1 & $\begin{array}{l}\text { Was the research question or objective in this } \\
\text { paper clearly stated? }\end{array}$ & $\begin{array}{l}\text { "Assessment of MNS in } \\
\text { schizophrenia spectrum disorders } \\
\text { patients" is clearly defined as an } \\
\text { objective. }\end{array}$ \\
\hline \#2 & $\begin{array}{l}\text { Was the study population clearly specified and } \\
\text { defined? }\end{array}$ & $\begin{array}{l}\text { Baseline characteristics of the study } \\
\text { population, specifically the definite } \\
\text { diagnosis of schizophrenia spectrum } \\
\text { disorders are stated. }\end{array}$ \\
\hline \#3 & $\begin{array}{l}\text { Was the participation rate of eligible persons at } \\
\text { least } 50 \% \text { ? }\end{array}$ & $\begin{array}{l}\text { More than } 50 \% \text { of the eligible } \\
\text { population participated in the study. }\end{array}$ \\
\hline \#4 & $\begin{array}{l}\text { Were all the subjects selected or recruited from } \\
\text { the same or similar populations (including the } \\
\text { same time period)? Were inclusion and exclusion } \\
\text { criteria for being in the study prespecified and } \\
\text { applied uniformly to all participants? }\end{array}$ & $\begin{array}{l}\text { Inclusion and exclusion criteria were } \\
\text { developed before recruitment or } \\
\text { selection of the study population and } \\
\text { the same underlying criteria were } \\
\text { used for all of the subjects involved. }\end{array}$ \\
\hline \#5 & $\begin{array}{l}\text { Was a sample size justification, power } \\
\text { description, or variance and effect estimates } \\
\text { provided? }\end{array}$ & $\begin{array}{l}\text { No: Most of our targeted studies will } \\
\text { be explanatory in nature. }\end{array}$ \\
\hline \#6 & $\begin{array}{l}\text { For the analyses in this paper, were the } \\
\text { exposure(s) of interest measured prior to the } \\
\text { outcome(s) being measured? }\end{array}$ & $\begin{array}{l}\text { In cohort studies, MNS evaluation } \\
\text { was done after the diagnosis. } \\
\text { In cross-sectional studies, the answer } \\
\text { is "No". }\end{array}$ \\
\hline \#7 & $\begin{array}{l}\text { Was the timeframe sufficient so that one could } \\
\text { reasonably expect to see an association between } \\
\text { exposure and outcome if it existed? }\end{array}$ & $\begin{array}{l}\text { In cohort studies, at least one year is } \\
\text { passed after the diagnosis when } \\
\text { MNS is evaluated. } \\
\text { In cross-sectional studies, the answer } \\
\text { is "No". }\end{array}$ \\
\hline \#8 & $\begin{array}{l}\text { For exposures that can vary in amount or level, } \\
\text { did the study examine different levels of the } \\
\text { exposure as related to the outcome (e.g., } \\
\text { categories of exposure, or exposure measured as } \\
\text { continuous variable)? }\end{array}$ & $\begin{array}{l}\text { Not applicable: Our exposure } \\
\text { (diagnosis of the disease) does not } \\
\text { have variation in amount. }\end{array}$ \\
\hline \#9 & $\begin{array}{l}\text { Were the exposure measures (independent } \\
\text { variables) clearly defined, valid, reliable, and } \\
\text { implemented consistently across all study } \\
\text { participants? }\end{array}$ & $\begin{array}{l}\text { Diagnosis of the disease was made } \\
\text { by a trained physician based on the } \\
\text { DSM or ICD criteria. }\end{array}$ \\
\hline$\# 10$ & $\begin{array}{l}\text { Was the exposure(s) assessed more than once } \\
\text { over time? }\end{array}$ & $\begin{array}{l}\text { Not applicable: Multiple times of } \\
\text { diagnosis of the disease I not } \\
\text { necessary. }\end{array}$ \\
\hline$\# 11$ & $\begin{array}{l}\text { Were the outcome measures (dependent } \\
\text { variables) clearly defined, valid, reliable, and } \\
\text { implemented consistently across all study } \\
\text { participants? }\end{array}$ & $\begin{array}{l}\text { It is clearly stated in the study that } \\
\text { they evaluate MNS. }\end{array}$ \\
\hline \#12 & Were the outcome assessors blinded to the & Assessors were blinded to the \\
\hline
\end{tabular}




\begin{tabular}{|c|c|c|}
\hline & exposure status of participants? & diagnosis of patients. \\
\hline \multirow[t]{2}{*}{ \#13 } & Was loss to follow-up after baseline $20 \%$ or less? & $\begin{array}{l}\text { In cohort studies, loss to follow-up } \\
\text { was less than } 20 \% \text {. }\end{array}$ \\
\hline & & $\begin{array}{l}\text { In cross-sectional the answer is "Not } \\
\text { applicable". }\end{array}$ \\
\hline \#14 & $\begin{array}{l}\text { Were key potential confounding variables } \\
\text { measured and adjusted statistically for their } \\
\text { impact on the relationship between exposure(s) } \\
\text { and outcome(s)? }\end{array}$ & $\begin{array}{l}\text { Regression methods were used for } \\
\text { adjustment for baseline differences. }\end{array}$ \\
\hline
\end{tabular}

\section{Declarations}

\section{Contributions}

$\mathrm{AV}$ is the leading author for protocol development, analyses, and dissemination. $\mathrm{AV}$ is also the first reviewer and the corresponding author. All authors will contribute to data interpretation and article drafts.

\section{Amendments}

Important protocol amendments post registration will be recorded and included in dissemination.

\section{Support}

No sources of support or funding were provided for this review.

\section{Conflicts of interest}

All authors declare there are no conflicts of interest regarding this study or its possible results.

\section{References}

1. Moher D, Shamseer L, Clarke M, Ghersi D, Liberati A, Petticrew M, et al. Preferred reporting items for systematic review and meta-analysis protocols (PRISMA-P) 2015 statement. Systematic reviews. 2015;4(1):1-9.

2. McGrath J, Saha S, Chant D, Welham J. Schizophrenia: a concise overview of incidence, prevalence, and mortality. Epidemiologic reviews. 2008;30(1):67-76.

3. Bora E, Yucel M, Pantelis C. Theory of mind impairment in schizophrenia: meta-analysis. Schizophrenia research. 2009;109(1-3):1-9.

4. Gold JM. Cognitive deficits as treatment targets in schizophrenia. Schizophrenia research. 2004;72(1):21-8. 
5. Green MF, Harvey PD. Cognition in schizophrenia: Past, present, and future. Schizophrenia Research: Cognition. 2014;1(1):e1-e9.

6. Organization WH. International classification of diseases 11th Revision [CID-11]. Recuperado de https://icd. who. int; 2018.

7. Rizzolatti G, Craighero L. The mirror-neuron system. Annu Rev Neurosci. 2004;27:169-92.

8. Rizzolatti G, Sinigaglia $\mathrm{C}$. The functional role of the parieto-frontal mirror circuit: interpretations and misinterpretations. Nature reviews neuroscience. 2010;11(4):264-74.

9. Di Pellegrino G, Fadiga L, Fogassi L, Gallese V, Rizzolatti G. Understanding motor events: a neurophysiological study. Experimental brain research. 1992;91(1):176-80.

10. Gallese V, Fadiga L, Fogassi L, Rizzolatti G. Action recognition in the premotor cortex. Brain. 1996;119(2):593-609.

11. Rizzolatti G, Fogassi L, Gallese V. Neurophysiological mechanisms underlying the understanding and imitation of action. Nature reviews neuroscience. 2001;2(9):661-70.

12. Umilta MA, Kohler E, Gallese V, Fogassi L, Fadiga L, Keysers C, et al. I know what you are doing: A neurophysiological study. Neuron. 2001;31(1):155-65.

13. Dinstein I, Hasson U, Rubin N, Heeger DJ. Brain areas selective for both observed and executed movements. Journal of neurophysiology. 2007;98(3):1415-27.

14. Gazzola V, Keysers C. The observation and execution of actions share motor and somatosensory voxels in all tested subjects: single-subject analyses of unsmoothed fMRI data. Cerebral cortex. 2009;19(6):1239-55.

15. Kilner JM, Neal A, Weiskopf N, Friston KJ, Frith CD. Evidence of mirror neurons in human inferior frontal gyrus. Journal of Neuroscience. 2009;29(32):10153-9.

16. Rizzolatti G, Fadiga L, Gallese V, Fogassi L. Premotor cortex and the recognition of motor actions. Cognitive brain research. 1996;3(2):131-41.

17. Decety J, Grezes J, Costes N, Perani D, Jeannerod M, Procyk E, et al. Brain activity during observation of actions. Influence of action content and subject's strategy. Brain: a journal of neurology. 1997;120(10):1763-77.

18. Buccino G, Binkofski F, Fink GR, Fadiga L, Fogassi L, Gallese V, et al. Action observation activates premotor and parietal areas in a somatotopic manner: an fMRI study. European journal of neuroscience. 2001;13(2):400-4.

19. Grèzes J, Armony JL, Rowe J, Passingham RE. Activations related to "mirror" and "canonical" neurones in the human brain: an fMRI study. Neuroimage. 2003;18(4):928-37.

20. Chong TTJ, Cunnington R, Williams MA, Kanwisher N, Mattingley JB. fMRI adaptation reveals mirror neurons in human inferior parietal cortex. Current biology. 2008;18(20):1576-80.

21. Molenberghs $P$, Cunnington R, Mattingley JB. Brain regions with mirror properties: a meta-analysis of 125 human fMRI studies. Neuroscience \& Biobehavioral Reviews. 2012;36(1):341-9. 
22. Grezes J, Decety J. Functional anatomy of execution, mental simulation, observation, and verb generation of actions: a meta-analysis. Human brain mapping. 2001;12(1):1-19.

23. Rizzolatti G, Fabbri-Destro M. The mirror system and its role in social cognition. Current opinion in neurobiology. 2008;18(2):179-84.

24. Bonini L, Rozzi S, Serventi FU, Simone L, Ferrari PF, Fogassi L. Ventral premotor and inferior parietal cortices make distinct contribution to action organization and intention understanding. Cerebral Cortex. 2010;20(6):1372-85.

25. Fogassi L, Ferrari PF, Gesierich B, Rozzi S, Chersi F, Rizzolatti G. Parietal lobe: from action organization to intention understanding. Science. 2005;308(5722):662-7.

26. Brass $M$, Heyes $C$. Imitation: is cognitive neuroscience solving the correspondence problem? Trends in cognitive sciences. 2005;9(10):489-95.

27. Buccino G, Vogt S, Ritzl A, Fink GR, Zilles K, Freund H-J, et al. Neural circuits underlying imitation learning of hand actions: an event-related fMRI study. Neuron. 2004;42(2):323-34.

28. Rizzolatti G, Sinigaglia C. The mirror mechanism: a basic principle of brain function. Nature Reviews Neuroscience. 2016;17(12):757-.

29. Bonini L. The extended mirror neuron network: Anatomy, origin, and functions. The Neuroscientist. 2017;23(1):56-67.

30. Caspers S, Zilles K, Laird AR, Eickhoff SB. ALE meta-analysis of action observation and imitation in the human brain. Neuroimage. 2010;50(3):1148-67.

31. lacoboni $M$, Dapretto $M$. The mirror neuron system and the consequences of its dysfunction. Nature Reviews Neuroscience. 2006;7(12):942-51.

32. Hamilton AF. The mirror neuron system contributes to social responding. Cortex. 2013;49(10):2957-9.

33. Marshall PJ, Meltzoff AN. Neural mirroring mechanisms and imitation in human infants. Philosophical Transactions of the Royal Society B: Biological Sciences. 2014;369(1644):20130620-.

34. Carr L, lacoboni M, Dubeau M-C, Mazziotta JC, Lenzi GL. Neural mechanisms of empathy in humans: a relay from neural systems for imitation to limbic areas. Proceedings of the national Academy of Sciences. 2003;100(9):5497-502.

35. Iacoboni M. Imitation, empathy, and mirror neurons. Annual review of psychology. 2009;60:653-70.

36. Théoret $H$, Pascual-Leone A. Language acquisition: Do as you hear. Current Biology. 2002;12(21):R736-R7.

37. Wolf NS, Gales ME, Shane E, Shane M. The developmental trajectory from amodal perception to empathy and communication: The role of mirror neurons in this process. Psychoanalytic Inquiry. 2001;21(1):94-112.

38. Green MF, Horan WP, Lee J. Social cognition in schizophrenia. Nature Reviews Neuroscience. 2015;16(10):620-31.

39. Reviews Cf, Dissemination. CRD's guidance for undertaking reviews in healthcare: York Publ. Services; 2009. 
40. Stroup DF, Berlin JA, Morton SC, Olkin I, Williamson GD, Rennie D, et al. Meta-analysis of observational studies in epidemiology: a proposal for reporting. Jama. 2000;283(15):2008-12.

41. Cooke A, Smith D, Booth A. Beyond PICO: the SPIDER tool for qualitative evidence synthesis. Qualitative health research. 2012;22(10):1435-43.

42. Organization WH. INTERNATIONAL CLASSIFICATION OF DISEASES-NINTH REVISION (ICD-9). Weekly Epidemiological Record= Relevé épidémiologique hebdomadaire. 1988;63(45):343-4.

43. Organization WH. International classification of diseases, 10th revision. Geneva: World Health Organization; Available: http. icd9cm chrisendres com/ Accessed July 8th. 2008.

44. Association AP. DSM-IV: Diagnostic and statistical manual of mental disorders. 1980.

45. Association AP. Diagnostic and statistical manual of mental disorders (DSM-5®): American Psychiatric Pub; 2013.

46. Hupe M. EndNote X9. Journal of Electronic Resources in Medical Libraries. 2019;16(3-4):117-9.

47. National Heart L, Institute B. Quality assessment tool for observational cohort and cross-sectional studies. Bethesda: National Institutes of Health, Department of Health and Human Services. 2014:103-11.

48. Team RC. R: A language and environment for statistical computing. 2013.

49. Higgins JPT, Thomas J, Chandler J, Cumpston M, Li T, Page MJ, et al. Cochrane handbook for systematic reviews of interventions: John Wiley \& Sons; 2019.

50. Borenstein M, Hedges LV, Higgins JPT, Rothstein HR. Introduction to meta-analysis: John Wiley \& Sons; 2011.

51. Lewin S, Glenton C, Munthe-Kaas H, Carlsen B, Colvin CJ, Gülmezoglu M, et al. Using qualitative evidence in decision making for health and social interventions: an approach to assess confidence in findings from qualitative evidence syntheses (GRADE-CERQual). PLoS Med. 2015;12(10):e1001895. 